\title{
HIV Status Disclosure to Sexual Partner(s) and Associated Factors Among Young Adults, A Mixed Methods Study
}

\author{
Tafadzwa Dzinamarira ${ }^{1,3, *}$, Martha Ndapandula Hatutale ${ }^{2}$, Hilda Namwenyo Ndadi ${ }^{2}$, \\ Moreblessing Chipo Mashora ${ }^{2}$, Saara Penehafo Shatumbu ${ }^{2}$ \\ ${ }^{1}$ School of Nursing, International University of Management, Windhoek, Namibia \\ ${ }^{2}$ Technical Department, Namibia Institute of Pathology, Windhoek, Namibia \\ ${ }^{3}$ Global Programs for Research and Training, University of California, San Francisco, USA
}

Email address:

tafadzwa.dzinamarira@ucglobalprograms.org (T. Dzinamarira)

${ }^{*}$ Corresponding author

To cite this article:

Tafadzwa Dzinamarira, Martha Ndapandula Hatutale, Hilda Namwenyo Ndadi, Moreblessing Chipo Mashora, Saara Penehafo Shatumbu. HIV Status Disclosure to Sexual Partner(s) and Associated Factors Among Young Adults, A Mixed Methods Study. International Journal of HIV/AIDS Prevention, Education and Behavioural Science. Vol. 5, No. 1, 2019, pp. 47-51. doi: 10.11648/j.ijhpebs.20190501.16

Received: February 24, 2019; Accepted: March 30, 2019; Published: May 15, 2019

\begin{abstract}
With a documented HIV prevalence among adults of $12.6 \%$ with disparities by sex; $15.7 \%$ and $9.3 \%$ among women and men respectively, Namibia is one of the first countries reported to be nearing epidemic control for HIV. Namibia has been very successful in ensuring universal treatment access for people living with HIV. For prevention interventions, the importance of HIV status disclosure among HIV-infected individuals, particularly to their sexual partners cannot be over emphasized. We used a mixed methods approach on a combined sample of 185 HIV positive young adults attending comprehensive care departments at selected referral hospitals in Namibia. For quantitative data, the chi-square test and multiple regression analysis were employed for data analysis. Qualitative data was transcribed, coded and analysed to build themes in line with study objectives. Of the 185 participants; only 35 were included in the qualitative component of the study with stratification by gender and age. Multiple logistic regression found HIV post-test counselling plus age were the only factors associated with disclosure status whilst adjusting for other study variables. Three major themes were built from the qualitative data. Theme one showed fear to be an important barrier to disclosure (fear of being rejected and violence). Theme two showed revealed lack of knowledge on importance of disclosure to sexual partner if not married or cohabitating. Theme three showed one main motivation for disclosure was having been tested together or discussed with sexual partner prior to testing. The importance of HIV post-test counselling remains an important factor affecting HIV status disclosure. In the advent of new HIV interventions such as HIV self-testing, findings from our study may be used to guide policies for these new interventions and strengthen post-test counselling to ensure HIV status disclosure to sexual partner(s). Health education on importance of testing as couples or with sexual partner needs to be strengthened.
\end{abstract}

Keywords: HIV Status Disclosure, Sexual Partner, Young Adults

\section{Introduction}

Human Immunodeficiency Virus (HIV) remains a public health problem, accounting for more than 1.8 million incidents yearly of which most incidents are common in resource poor countries [1]. For Namibia, The Joint United Nations Programme on HIV and AIDS (UNAIDS) reported that there were $260,000(240,000-280,000)$ people living with HIV (PLWH) in Namibia, an estimated 5,100 (3,500 -
$9,100)$ deaths, and $53,000(38,000-93,000)$ orphans due to HIV [1].

Several studies have revealed that up to one third of individuals diagnosed with HIV infection continue to have unprotected sex, at times without informing their sexual partners, who may be of negative or unknown sero-status $[2$, 3]. The prevention and control of HIV infection depends on the success of strategies to prevent new infections and treat currently infected individuals. HIV status disclosure is an 
important component of HIV prevention as it may motivate partners to also want to know their HIV status, reduce HIV related risk sexual behavior and ultimately reduce HIV transmission [4].

Bachanas et al. described the frequency of and factors associated with disclosure, knowledge of partner's HIV status, and consistent condom use among 3538 HIV-positive patients attending eighteen HIV care and treatment clinics in Kenya, Namibia, and Tanzania [5]. In their study, the majority $(80 \%)$ had disclosed their HIV status to their partners, $64 \%$ knew their partner's HIV status, and $77 \%$ reported consistent condom use. Of those who knew their partner's status, $18 \%$ reported their partner was HIV negative [5].

The same study showed that compared to men, women were significantly less likely to report disclosing their HIV status to their sex partner(s), to knowing their partner's HIV status, and to using condoms consistently with HIV-negative partners [5]. Other factors negatively associated with consistent condom use included nondisclosure, alcohol use, reporting a casual sex partner, and desiring a pregnancy [5]. Unsafe sex is believed to be the second most important risk factor for HIV acquisition, and death in the poorest countries of the world. It is not having sex, but having unprotected sex rather, with a partner whose status is not known or who is HIV positive that places people at serious risk of HIV infection [6]. For prevention interventions, the importance of HIV status disclosure among HIV-infected individuals, particularly to their sexual partners cannot be over emphasized.

\section{Materials and Methods}

\subsection{Study Design}

For this study, we employed a mixed methods approach on a calculated sample size of 185 young adults aged between 18 to 35 years. Ethical approval was obtained from the Ministry of Health and Social Services, Namibia.

\subsection{Data Collection and Statistical Analysis}

Quantitative data was collected using a structured, pretested self- administered questionnaire. Participant's responses were captured on paper, transferred onto Open Data Kit and electronically processed using STATA (StataCorp, Version 15) statistical package. Results were presented numerically using tables. Relative importance index (RII) values were calculated to assess the disclosure of HIV status to sexual partners and the independent variables based on responses on an ordinal scale using Eq. 1.

$$
\mathrm{RII}=\sum \mathrm{Qi} / \mathrm{NS}
$$

where $\mathrm{Pi}$ is the respondent's rating on the ordinal scale for question $\mathrm{i}$ of the indicator, $\mathrm{N}$ is the number of questions asked per indicator, $\mathrm{S}$ is the highest possible score on the ordinal scale. Means, median or frequencies were used in reporting results where appropriate.
The chi-square test or student's t-test was used to determine association between individual study variable and the disclosure of HIV status to sexual partners. During bivariate analysis step several levels of categorical variables were combined to form new categories, RIIs and other continuous variables were categorized by means of the median value cut-off. Multiple regression analysis was used to determine factors associated disclosure of HIV status to sexual partner(s) RII score while adjusting for other study variables. Stepwise approach was used to determine independent variables necessary for inclusion in the final model. Only variables with $(\mathrm{p}<0.25)$ in bivariate analysis were included for analysis in multiple regression. Statistical conclusions were reached at $5 \%$ level of significance.

A subset of the study participants, 35, participated in the qualitative study component of the study with stratification by gender and age. Data collection was through open interviews. All tape-recorded interviews were transcribed verbatim. Observation notes were taken during each interview. The text document of the note and the transcribed information were coded to identify major themes. Finally, an overall interpretation was done about how thematic areas related to one another, explaining how the various concepts related to the study question. We employed NVivo version 12 software (QSR International Pty Ltd. Version 10, 2012) for qualitative data analysis.

\section{Results}

Table 1. Demographics.

\begin{tabular}{ll}
\hline Variable & Value \\
\hline 1. Gender, n (\%) & $106(57)$ \\
Male & $79(43)$ \\
Female & $26.4(5.3)$ \\
2. Age in years, mean (SD) & \\
3. Highest level of education, n (\%) & $12(6)$ \\
Primary & $72(54)$ \\
Secondary & $99(39)$ \\
Tertiary & $2(1)$ \\
Did not attend school & \\
4. Source of financial income, n (\%) & $80(43)$ \\
Self employed & $58(32)$ \\
Professional & $47(25)$ \\
Unemployed & \\
5. Location of residence, n (\%) & $103(56)$ \\
Windhoek urban & $71(38)$ \\
Windhoek rural & $11(6)$ \\
Other & \\
6. Ethnicity, n (\%) & $8(4)$ \\
Colored & $21(11)$ \\
Damara & $1(0.5)$ \\
Himba & $14(7.5)$ \\
Kavango & $2(1)$ \\
Nama & $98(53)$ \\
Ovambo & $30(16)$ \\
Herero & $11(6)$ \\
7. Marital status, n $(\%)$ & $35(19)$ \\
Cohabiting & $21(11.5)$ \\
Married & $1(0.5)$ \\
Separated & $128(69)$ \\
\hline
\end{tabular}


A total of 185 subjects aged $18-35$ were recruited in the study. Detailed information about the demographic characteristics of the study participants are available in Table 1. Of the participants; $43 \%$ were females, the average age (SD) was 26.4 years (5.3), 6\% were educated up to primary school only, $25 \%$ were unemployed, $56 \%$ reported residence in Windhoek urban area while $38 \%$ and $6 \%$ resided in Windhoek rural or other classification, $53 \%$ were of the Ovambo ethnicity while $47 \%$ were from other ethnical groupings, $69 \%$ were single while $11.5 \%$ and $19 \%$ were married and cohabiting respectively.

There was an association between age and having ever disclosed HIV status $(\mathrm{p}<0.001)$, those who disclosed had mean (SD) age of 28 (4.5) years while those who never disclosed are 25.2 (5.5) years old. There was an association between source of income and having ever disclosed HIV status $(\mathrm{p}=0.037)$, only $37 \%$ of self-employed disclosed, compared to $46 \%$ and $52 \%$ among those unemployed or professionals respectively. There was an association between ever paid or receive payment for sex and having ever disclosed HIV status $(p=0.010)$, only $33 \%$ of participants who transact for sex have disclosed their status compared to $52 \%$ among those who do not transact for sex. There was an association between ever received HIV post-test counselling and having ever disclosed HIV status $(\mathrm{p}<0.001)$, 87\% who had counselling before have disclosed their status compared to $5 \%$ among those who never got counselling.

There was an association between knowledge of HIV and having ever disclosed HIV status ( $\mathrm{p}=0.003$ ), 42\% participants knowledgeable have disclosed their status compared to $54 \%$ who had poor knowledge. Other factors tested for association with disclosure status were found to have no significant association with disclosure status. More detailed information is available in table 2 .

Table 2. Factors associated with HIV status disclosure by bivariate analysis.

\begin{tabular}{llll}
\hline \multirow{2}{*}{ Variable } & \multicolumn{2}{l}{ Ever disclosed } & \multirow{2}{*}{ p-value } \\
\cline { 2 - 3 } & No & Yes & \\
Gender, (\%) & & & \\
Female & $42(53)$ & $37(47)$ & 0.211 \\
Male & $62(58)$ & $44(42)$ & \\
Age in years, mean (SD) & $25.2(5.5)$ & $28.0(4.5)$ & $<0.001$ \\
Source of income, (\%) & & & \\
Self employed & $51(63)$ & $29(37)$ & 0.037 \\
Unemployed & $25(54)$ & $22(46)$ & \\
Professionals & $28(48)$ & $30(52)$ & \\
Marital status, n (\%) & & & \\
Single & $73(57)$ & $55(43)$ & 0.217 \\
Others & $30(52)$ & $27(47)$ & \\
Number of sexual partners ever had & & & \\
in life, n (\%) & & & \\
One & $19(46)$ & $22(54)$ & 0.226 \\
$\geq$ One & $75(52)$ & $69(48)$ & \\
Sexual preference, n (\%) & & & \\
Heterosexual & $70(53)$ & $162(47)$ & 0.132 \\
Others & $31(60)$ & $22(40)$ & \\
Ever paid or received payment for & & & \\
sex, n (\%) & & & \\
No & $68(48)$ & $72(52)$ & 0.010 \\
Yes & $30(67)$ & $15(33)$ & \\
Ever had HIV post-test counselling & & & \\
\hline
\end{tabular}

\begin{tabular}{llll}
\hline \multirow{2}{*}{ Variable } & \multicolumn{2}{l}{ Ever disclosed } & \multirow{2}{*}{ p-value } \\
\cline { 2 - 3 } & No & Yes & \\
\hline done, n (\%) & & & \\
No & $89(95)$ & $5(5)$ & $<0.001$ \\
Yes & $12(13)$ & $79(87)$ & \\
Ever been taught or heard about sex & & & \\
education, n (\%) & & & \\
No & $79(52)$ & $73(47)$ & 0.089 \\
Yes & $21(64)$ & $12(36)$ & \\
Knowledge of HIV, n (\%) & & & \\
Poor $(<8$ out of 10 score) & $8(35)$ & $14(65)$ & 0.003 \\
Good $(>8$ out of 10 score) & $95(58)$ & $68(42)$ & \\
\hline
\end{tabular}

Multiple logistic regression was used to determine factors associated with disclosure status whilst adjusting for other study variables. It was found that having ever gone for HIV counselling and testing plus age were the only factors associated with disclosure status. The odds of having disclosed the HIV status was 12.5 times among participants who have ever gone for HIV counselling and testing than those who have never accessed HIV counselling and testing $(p<0.001)$, whilst adjusting for other study variables. It was also found that a year increase in age would increase the odds of disclosure by 1.07 times $(\mathrm{p}=0.042)$, whilst adjusting for other study variables.

Table 3. Factors associated with HIV status disclosure by multivariate logistic regression analysis.

\begin{tabular}{lll}
\hline Variable & Odd Ratio (95\% CI) & p-value \\
\hline $\begin{array}{l}\text { Ever had HIV counselling and } \\
\text { testing done, } \mathrm{n}(\%)\end{array}$ & & \\
Yes & $12.5(5.7-27.7)$ & $<0.001$ \\
Age in years, mean (SD) & $1.07(1.0-1.15)$ & 0.042 \\
\hline
\end{tabular}

In the interviews with young adults living with HIV aged 15 - 35 attending comprehensive care departments at selected referral hospitals in Windhoek, Namibia, participants described a combination of barrier and motivational factors that influenced their disclosure. What follows is a presentation of these factors described by the 35 participants we interviewed. Three major themes were built from the qualitative data. Theme one showed fear to be an important barrier to disclosure (fear of being rejected and violence).

"........ if I tell him this, what do you think would happen to me. I am afraid. I will keep it for as long as I can. HIV is a serious infection. My partner would leave me. I know that." 29-year-old female. Has known status for two years.

"My husband is an abusive man. I cannot tell him this and expect to be ok. He will beat me." 31-year-old female. Has known status for three years.

Theme two showed revealed lack of knowledge on importance of disclosure to sexual partner if not married or cohabitating.

"My main reason is I know if I tell her she will just leave me and move on to the next person. In fact, what will stop her from telling everyone I know about my status. We are not married. She is just a girlfriend and a girlfriend can wake up gone. I cannot tell her." 28-year-old male. Has known status for less than a year.

"We are not married. He has a wife. I don't have to tell him. If it was marriage, I would have to disclose to my 
husband but he is not." 33-year-old female. Has known status for five years.

Theme three showed one main motivation for disclosure was having been tested together or discussed with sexual partner prior to testing.

"...... after we got the results back, we went back home and discussed out results. I informed her that I had tested positive and she was devastated. We had been married for less than a year. She has now accepted it. It was tough but we are still together. My wife is HIV negative." 28-year-old male. Has known status for two years.

\section{Discussion}

We show HIV post-test counselling to be associated with HIV status disclosure. This conforms with other findings from previous studies by Kadowa and Nuwaha in Uganda [7], Deribe et al. in Ethiopia [8], Ogoina et al. in Nigeria [9] and Idindili et al. in Tanzania [10]. Obermeyer et al. reviewed facilitators of HIV status disclosure across diverse settings and their findings suggested that structural changes, including making more services available, could facilitate HIV disclosure as much as individual approaches and counseling do [11]. In the same light, findings from $\mathrm{Vu}$ et al. study in Cape Town, South Africa supported a positive prevention strategy that emphasizes increased access to ART, and behavioral interventions to reduce casual sex partnerships for persons who are HIV-positive [12]. Vu et al. go on to highlight that mitigating the influence of HIV stigma on HIV status disclosure particularly within steady sex partnerships is also important and may be accomplished through individual and couple counseling [12]. With Provider Initiated HIV testing at health facilities it has been easier to ensure post-test counselling. However, In the advent of new intervention such as HIV self-testing, policy makers need to craft well strategies that ensure HIV post-test counselling for individuals who perform HIV self-testing.

This study showed counselling to be strongly related to HIV status disclosure. This is however not in tandem with Sehosa and Peltzer who in their study found only social support to be significantly related to HIV status disclosure [13]. In their study, the sample consisted at the post-test counselling exit interview of 55 participants (41 women and 14 men) who tested HIV-positive conveniently selected from a rural hospital and at five months' follow-up at their homes. Results indicated that most patients had an HIV test because of medical reasons. At follow-up only $36 \%$ had disclosed their HIV status and half of the participants had had sex without condoms in the past three weeks [13]. Social support was found to be significantly related to disclosure of HIV status, while counselling context and content and counselling satisfaction were not related with HIV disclosure [13]. This finding indicated that counselling needed to be improved regarding partner notification [13]. Our findings show that there may have been good progress in improving counselling messages to do with partner notification.

Our findings suggest age may be an important predictor of
HIV status disclosure to sexual partner. This finding was similar to other studies $[12,14]$ which revealed that age, occupation, knowledge on HIV transmission, and content of discussion about HIV/AIDS were potential predictors for respondents to disclose HIV status.

Results from the exploratory part of our study revealed three themes. Theme one showed fear to be an important barrier to disclosure (fear of being rejected and violence). Theme two showed revealed lack of knowledge on importance of disclosure to sexual partner if not married or cohabitating. Theme three showed one main motivation for disclosure was having been tested together or discussed with sexual partner prior to testing. Similar findings have been observed in earlier studies [15] [18]. However, ignorance on importance of disclosure to sexual partner if not married or cohabitating shows a need to strengthen counselling messages and health education given to PLWH on their routine ARV clinic appointments.

\section{Conclusion}

Non-disclosure of HIV serostatus is a public health concern with serious implications for efforts toward HIV epidemic control. Our findings imply that in order to increase rates of HIV status disclosure to sexual among young adults, special emphasis should be made to support and counsel clients who test HIV positive. Issues of fear of rejection still exist among HIV positive Namibian young adults. There is a need to strengthen health education efforts for both the infected and affected. We recommend further studies with no age restrictions to fully understand the barriers and facilitators to HIV status disclosure among PLWH.

\section{Competing Interests}

The authors declare that they have no competing interests which may have inappropriately influenced them in writing this article.

\section{Acknowledgements}

We extend our gratitude to Messrs Vusumuzi Maliwa and Bernard Ngara for statistical input.

\section{References}

[1] UN Joint Programme on HIV/AIDS, UNAIDS. (2016). Global AIDS Update. Retrieved from http://www.refworld.org/docid/574e8d394.html

[2] Centers for Disease Control and Prevention. Revised guidelines for HIV counseling, testing and referral. MMWR Recomm Rep. 2001 Nov 9; 50(RR-19):1-57. quiz CE1-19a1CE6-19a1.

[3] Dan C, Ron S, Rebecca C, James C. San Francisco: AIDS Research Institute University of California; Disclosure of HIV status to sexual partners among a random sample of American seropositives. Available on: http://caps.ucsf.edu/uploads/pubs/presentations/pdf/ciccarone.pdf. 
[4] Simbayi LC, Kalichman SC, Strebel A, et al. (2007) Disclosure of HIV status to sex partners and sexual risk behaviours among HIV-positive men and women, Cape Town, South Africa. Sex Transm Infect. Feb; 83(1):29-34.

[5] Bachanas P, Medley A, Pals S, Kidder D, Antelman G, Benech I, DeLuca N, Nuwagaba-Biribonwoha H, Muhenje O, Cherutich P, Kariuki P, Katuta F, Bukuku M and PwP Study Group, Disclosure, Knowledge of Partner Status, and Condom Use Among HIV-Positive Patients Attending Clinical Care in Tanzania, Kenya, and Namibia, AIDS Patient Care STDS. $2013 \mathrm{Jul} ; 2$ 27(7): 425-435.

[6] Shisana, O., Rehle, T., Simbayi, L. C., Zuma, K., Jooste, S., Pillay-van Wyk, V., Mbelle, N., Van Zyl, J., Parker, W., Zungu, N. P., Pezi, S. and the SABSSM III Implementation Team (2009). South African national HIV prevalence. incidence and communication survey 2008: A turning tide among teenagers? Cape Town: Human Sciences Research Council Press.

[7] Kadowa, I., \& Nuwaha, F. (2009). Factors influencing disclosure of HIV positive status in Mityana district of Uganda. African health sciences, 9(1), 26-33.

[8] Deribe B, Ebrahim J, Bush L (2018) Outcomes and Factors Affecting HIV Status Disclosure to Regular Sexual Partner among Women Attending Antiretroviral Treatment Clinic. J AIDS Clin Res 9: 760. doi: 10.4172/2155-6113.1000760

[9] Ogoina, D., Ikuabe, P., Ebuenyi, I., Harry, T., Inatimi, O., \& Chukwueke, O. (2015). Types and predictors of partner reactions to HIV status disclosure among HIV-infected adult Nigerians in a tertiary hospital in the Niger Delta. African health sciences, 15(1), 10-8.

[10] Idindili B, Selemani M, Bakar F, Thawer S. G, Gumi A, Mrisho M, Kahwa A. M and Julius J. Massaga J. J. (2015) Enhancing HIV status disclosure and partners' testing through counselling in Tanzania. Tanzania Journal of Health Research. $17: 3$.
[11] Obermeyer C. M, Baijal P and Pegurri E (2011). "Facilitating HIV Disclosure Across Diverse Settings: A Review". American Journal of Public Health 101, no. 6 pp. 1011-1023.

[12] Vu L, Andrinopoulos K, Mathews C, Chopra M, Kendall C, Eisele T. P. (2012) Disclosure of HIV status to sex partners among HIV-infected men and women in Cape Town, South Africa. AIDS and Behavior. 16(1):132-8.

[13] Sethosa E, Peltzer K. Evaluation of HIV counselling and testing, self-disclosure, social support and sexual behaviour change among a rural sample of HIV reactive patients in South Africa. Curationis. 2005; 28(1):29-41.

[14] Nöstlinger C, Bakeera-Kitaka S, Buyze J, Loos J, and Buvé A. (2015) "Factors influencing social self-disclosure among adolescents living with HIV in Eastern Africa," AIDS Care, vol. 27 , no. 1 , pp. $36-46$.

[15] Korner H. Negotiating cultures: disclosure of HIV-positive status among people from minority ethnic communities in Sydney. Cult Health Sex. 2007; 9(2):137-152.

[16] Klitzman RL, Kirshenbaum SB, Dodge B, et al. Intricacies and inter-relationships between HIV disclosure and HAART: a qualitative study. AIDS Care. 2004; 16(5):628-640.

[17] Lugalla JLP, Madihi CM, Sigalla HL, Mrutu NE, Yoder PS Social Context of Disclosing HIV Test Results: HIV Testing in Tanzania. Dar es Salaam, Tanzania: Centre for Strategic Research and Development and Macro International; 2008. DHS Qualitative Research Studies.

[18] Cusick L. The process of disclosing positive HIV status: findings from qualitative research. Cult Health Sex. 1999; 1(1):3-18. 